\title{
Band Gap Opening Induced by the Structural Periodicity in Epitaxial Graphene Buffer Layer
}

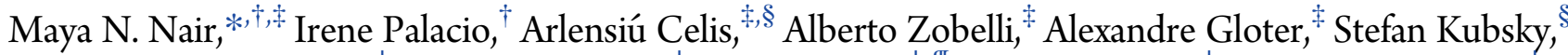 \\ Jean-Philippe Turmaud, ${ }^{\perp}$ Matthew Conrad, ${ }^{\perp}$ Claire Berger,, , I Walter de Heer, ${ }^{\perp}$ Edward H. Conrad,,${ }^{\prime}$ \\ Amina Taleb-Ibrahimi, ${ }^{\dagger}$ and Antonio Tejeda*,\$, \\ ${ }^{\dagger}$ UR1 CNRS/Synchrotron SOLEIL, Saint-Aubin, 91192 Gif sur Yvette, France \\ ${ }^{\ddagger}$ Laboratoire de Physique des Solides, CNRS, Univ. Paris-Sud, Universite Paris-Saclay, Bat. 510, F-91405 Orsay Cedex, France \\ Synchrotron SOLEIL, L'Orme des Merisiers, Saint-Aubin, 91192 Gif sur Yvette, France \\ ${ }^{\perp}$ School of Physics, The Georgia Institute of Technology, Atlanta, Georgia 30332-0430, United States \\ II \\ CNRS/Institut Neél, BP166, 38042 Grenoble, France
}

\begin{abstract}
The epitaxial graphene buffer layer on the Si face of hexagonal SiC shows a promising band gap, of which the precise origin remains to be understood, In this work, we correlate the electronic to the atomic structure of the buffer layer by combining angle resolved photoemission spectroscopy (ARPES), scanning tunneling microscopy (STM), and high-resolution scanning transmission electron microscopy (HR-STEM). We show that the band structure in the buffer has an electronic periodicity related to the structural periodicity observed in STM images and published X-ray diffraction. Our HR-STEM measurements show the bonding of the buffer layer to the $\mathrm{SiC}$ at specific locations separated by $1.5 \mathrm{~nm}$. This is consistent with the quasi $6 \times 6$ periodic corrugation observed in the STM images. The distance between buffer $\mathrm{C}$ and $\mathrm{SiC}$ is $1.9 \AA \AA$ in the bonded regions and up to $2.8 \AA$ in the decoupled regions, corresponding to a $0.9 \AA$ corrugation of the buffer layer. The decoupled regions are $\mathrm{sp}^{2}$ hybridized. Density functional tight binding (DFTB) calculations demonstrate the presence of a gap at the Dirac point everywhere in the buffer layer, even in the decoupled regions where the buffer layer has an atomic structure close to that of graphene. The surface periodicity also promotes band in the superperiodic Brillouin zone edges as seen by photoemission and confirmed by our calculations.
\end{abstract}

Graphene is a promising candidate for nanoelectronics because of its exceptional electronic properties. ${ }^{1-5}$

However, the absence of energy gap in this zero-gap semiconductor limits its applications in digital electronics. This problem has therefore motivated many different approaches to opening a gap ranging from chemical modification by doping or functionalization with atoms, molecules, or a substrate to patterning graphene into nanoribbons. ${ }^{1,6-17}$ In scalable nanoribbons, the precise control of the electronic properties requires edges controlled at an atomic level, which is still beyond the accuracy of current lithographic techniques. In fact, the observed transport gap for lithographic ribbons is due to localization effects caused by disordered ribbon edges rather than finite size effects. ${ }^{16}$ A particular interesting variety of graphene ribbons, ${ }^{17}$ grown on $\mathrm{SiC}$ facet templates, have their position defined by lithography prior to growth. Their ballistic transport ${ }^{1}$ indicates smooth edges, and the well-defined mini-ribbons that border the facets

show a band gap. ${ }^{18,19}$

The problem of tailoring the band gap in ribbons by controlling their width to nanometer dimensions could be avoided if a bottom-up graphene structure could be grown with an intrinsic band gap. Such a variety indeed exists on silicon carbide and is called the "buffer" layer. When $\mathrm{SiC}(0001)$ is annealed at temperatures below the optimal temperature for growing graphene, an atomic layer of carbon atoms with honeycomb structure appears. ${ }^{20,21}$ This buffer layer is semi-conducting with electronic properties different from those of graphene as shown in Figure 1a. Upon further annealing, another graphene layer

forms below the existing buffer layer so that the buffer converts to a conducting graphene layer with a new

buffer layer under it at the $\mathrm{SiC}$ interface. The top graphene layer exhibits the ideal dispersion of graphene $^{22-24}$ (Figure $1 \mathrm{~b}$ ) and is $\mathrm{n}$-doped with the Dirac point $-0.4 \mathrm{eV}$ below the Fermi level $\left(E_{\mathrm{F}}\right)$. The position of the Dirac point has been proposed to be caused by pinning $E_{\mathrm{F}}$ by interface states. ${ }^{25-27}$ This situation shifts graphene bands in energy with respect the buffer 

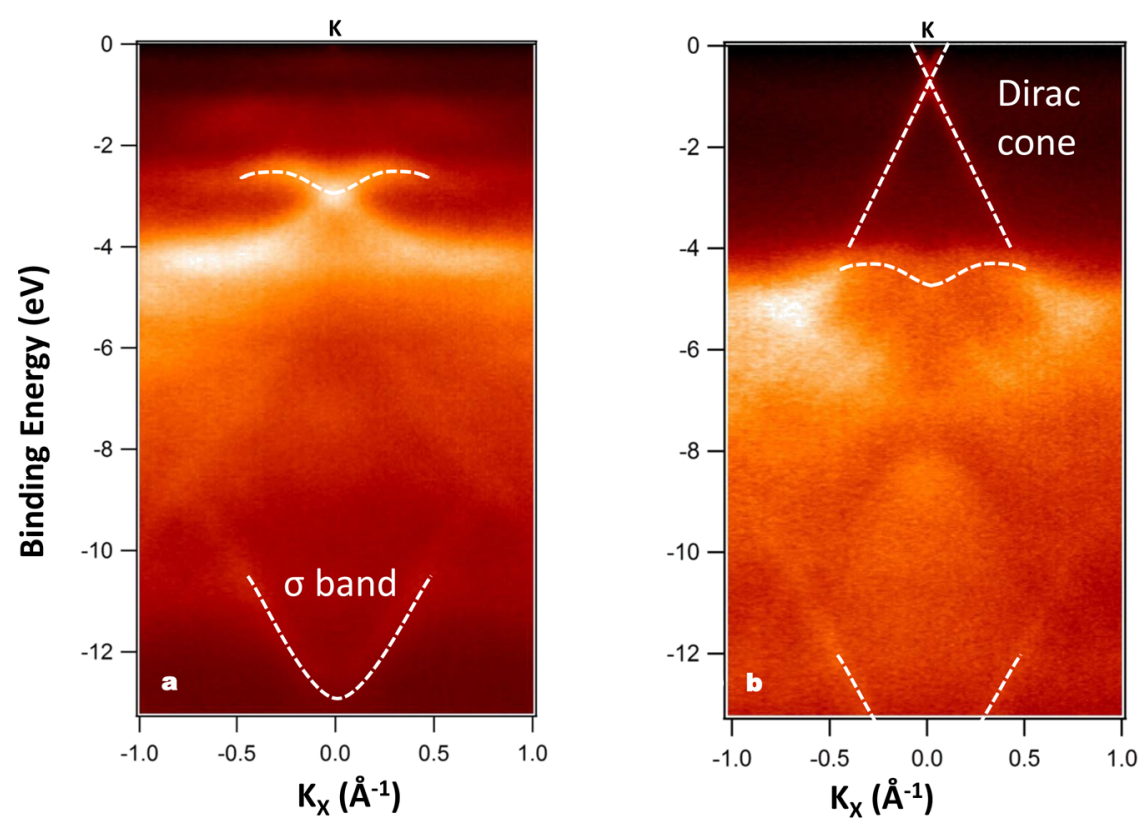

Figure 1. Electronic band structure through the $\mathrm{K}$ point in a direction perpendicular to $\Gamma \mathrm{K}$ (see Figure 2a for the Brillouin zone scheme) for (a) buffer layer and (b) graphene layer above the buffer layer. A band gap opening at the $\mathrm{K}$ point is clearly present in the buffer layer as its characteristic electronic states do not reach the Fermi level (panel a). The overall band structure is shifted toward higher binding energies when metallic graphene is grown on top of the semiconducting buffer layer (panel b). The shift can be interpreted as being due to a Schottky barrier between a metal and a p-doped semiconductor. Equivalent states in the buffer and in graphene system are indicated by dashed lines to highlight the shift in the electronic states upon graphene growth on top of the buffer layer. The graphene dispersion is marked in panel b by dashed lines.

layer. The buffer layer preserves the $\pi$ band of graphene although it becomes gapped by more than $0.5 \mathrm{eV}$, twice the previous value in epitaxial graphene samples. ${ }^{29}$

The buffer layer has been extensively studied. The atomic structure has been studied by STM, ${ }^{21,35-38,40,43}$ LEED, ${ }^{22,38,40,41}$ and other diffraction techniques ${ }^{30,43}$ and the electronic structure with ARPES ${ }^{22,23,28,29}$ and by STEM-EELS spectroscopy. ${ }^{19}$ Many calculations have also been performed to understand its structure and its electronic properties. $^{20,26,27,31-34}$ The buffer layer is observed as a $(6 \times 6)$ reconstruction in STM, ${ }^{35-38,40}$ while LEED pattern indicates a $(6 \sqrt{3} \times 6 \sqrt{3}) \mathrm{R} 30^{\circ 22,35,39-41}$ structure, but the apparent contradiction can be explained. ${ }^{27}$ From the electronic point of view, the buffer layer exhibits a band gap, as observed by ARPES $^{22,28}$ or by STS. ${ }^{42}$ This band gap could in principle appear due to a complete covalent bonding of the buffer layer with the substrate, suppressing the $\pi$ band of graphene and rendering the system insulating. However, such a global covalent bonding is hard to reconcile with the fact that hydrogen intercalation lifts the buffer from the substrate, decoupling it and converting it in quasi-free-standing monolayer graphene.

The current explanation for buffer layer band gap, while preserving the honeycomb lattice, is to attribute the gap to the formation of covalent $\mathrm{C}-\mathrm{Si}$ bonds between the buffer and the substrate on particular sites of the honeycomb lattice that are in near coincidence with $\mathrm{Si}$ atoms in the substrate surface. ${ }^{21,22,34}$ The gap's origin has also been suggested to be the result of a pseudo nanoribbon network of regions decoupled from the $\mathrm{SiC}^{28}$ Given the potential technological interest of having a gapped material entirely made of graphene, understanding the origin of the band gap in the buffer layer is important if the gap is to be used and controlled in relevant applications. Here we show that the electronic structure of the buffer layer is strongly modified by the observed $6 \times 6$ periodicity. Structural analysis is performed to quantify the presence of defects on the surface, the bonding to the substrate, and the correlation between the buffer's electronic and structural periodicities. For this we combine STM, cross-sectional-scanning transmission electron microscopy (X-STEM), and angle resolved photoemission spectroscopy (ARPES) together with density functional based tight binding (DFTB) calculations to give important insights into the nature of the buffer's observed band gap.

A well-suited technique to explore the formation of band gaps is angle-resolved photoemission that allows to map the band structure over the whole reciprocal space. Figure 2a shows a schematic of reciprocal space indicating the graphene Brillouin zone and its high symmetry points as well as a set of the smaller Brillouin zones of a $(6 \times 6)_{\mathrm{SiC}}$ incommensurate period recently observed by X-ray diffraction. ${ }^{43}$ The incom-mensurate density modulation has a fundamental wavelength of

$6(1+\delta) a_{\mathrm{SiC}}$ (where $a_{\mathrm{SiC}}$ is bulk SiC lattice constant and $\delta=$ $0.037)$. The band structure of the buffer layer measured along the $\Gamma \mathrm{KM}$ direction $\left(\mathrm{K}_{y}\right)$ of graphene (red arrow in Figure $2 \mathrm{a}$ ) is shown in Figure 2b. Figure 2c shows an ARPES cut through the $\mathrm{K}$ point in a direction perpendicular to $\Gamma \mathrm{K}, \mathrm{K}_{x}$ (blue arrow in

Figure 2a). The spectrum shows $\sigma$ bands in the buffer layer as expected from the presence of in-plane $\sigma$ bonds in the buffer honeycomb structure (Figure $2 b$ ). The spectra show no evidence of a metallic linear dispersing $\pi$ band either in the $\Gamma \mathrm{K}$ direction or in the perpendicular direction through the $\mathrm{K}$ point. A close up of the bands at the $\mathrm{K}$ point shows the gapped spectral feature $\varepsilon_{1}$ (Figure $2 \mathrm{~d}$ ) in agreement with ref 28 . Its spectral weight is distributed along a parabola, with a wide gap at the Dirac point of more than $0.4-0.5 \mathrm{eV}$ in the occupied part of the band structure (estimated from the energy of the first electronic state to $E_{\mathrm{F}}$ ), confirming the semiconducting properties of the buffer layer. The highest spectral weight in $\varepsilon_{1}$ is reached at the energies around $-0.4 \mathrm{eV}$ and $-1.4 \mathrm{eV}$, which correspond roughly to the binding energies of the $g_{1}$ and 

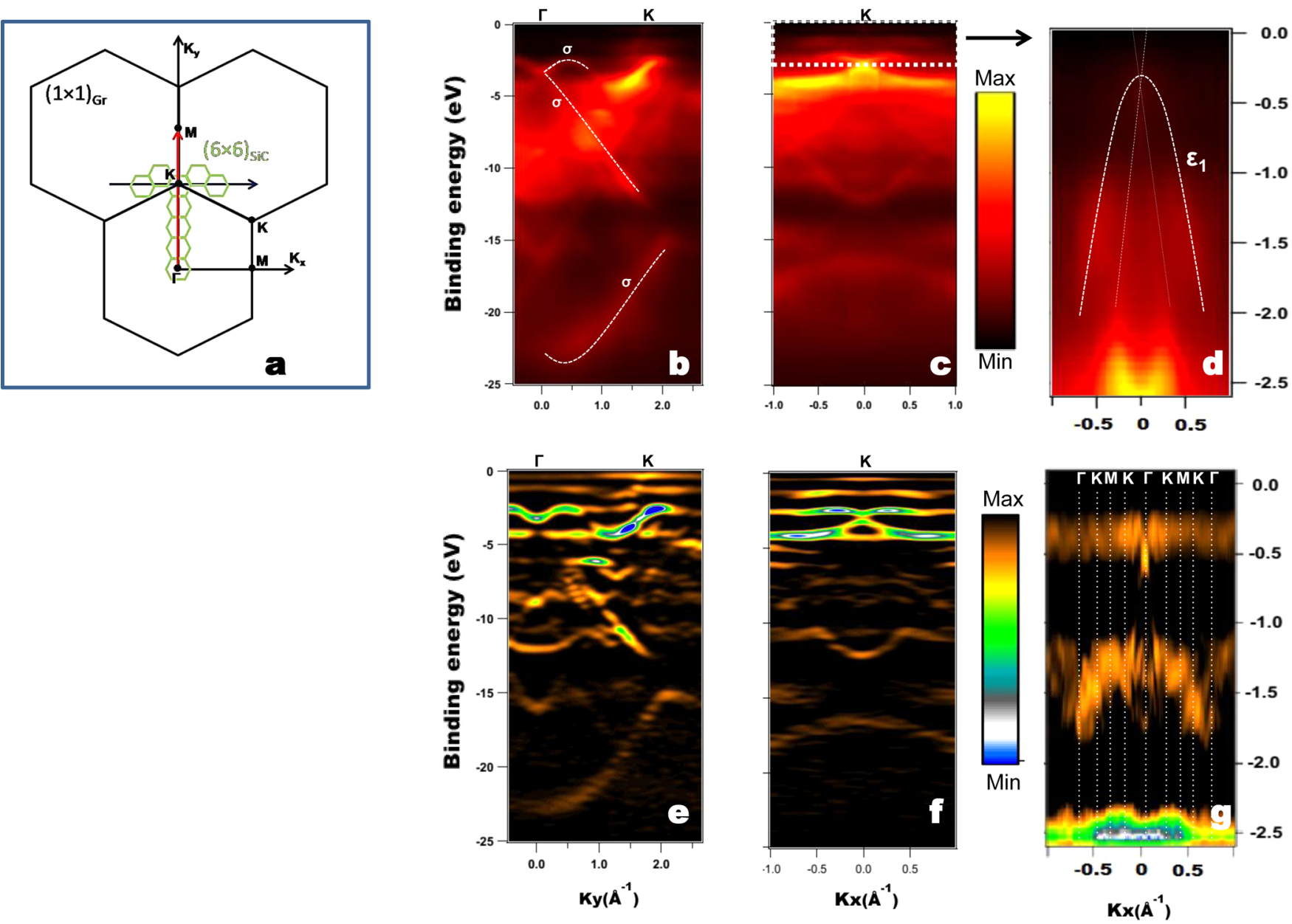

Figure 2. Electronic band structure of the buffer layer, with the binding energy relative to $E_{\mathrm{F}}=0$. (a) Scheme of the Brillouin zones of the system. The large black hexagon corresponds to graphene Brillouin zone. The small green hexagons correspond to a pseudo $(6 \times 6)_{\mathrm{SiC}}$. The red(blue) arrow corresponds to the ARPES scan directions in $b(c)$. (b) ARPES measurement along $\Gamma K$ direction showing the band gap at the $K$ point and the presence of the $\sigma$ band characteristic of in-plane bonds in graphene-like structures. (c) ARPES measurement through the $\mathrm{K}$ point in a direction perpendicular to ГKM. (d) Zoom of $c$ (from the dotted rectangle in c) showing the detail of the electronic structure of the buffer layer. A faint Dirac cone at a domain with graphene is also present, as indicated by the dashed lines crossing themselves at the Dirac point. A dotted parabola marks the spectral weight of the $\varepsilon 1$ spectral feature of the buffer layer. It has intensity maxima at approximately -0.4 and $-1.4 \mathrm{eV}$. (e-g) Second derivatives of $\mathrm{b}-\mathrm{d}$, respectively. A low intensity linear dispersion is also observed due to the presence of small domains of graphene. The high symmetry points of the pseudo $(6 \times 6)_{S i C}$ periodicity are indicated on the top of the panel $g$.

$\mathrm{g}_{2}$ states clearly observed in the earlier stages of growth and that have been previously observed in UHV-grown buffer layer. ${ }^{22}$ The second derivative of Figure $2 b-d$ is shown, respectively, in Figure $2 \mathrm{e}-\mathrm{g}$. This is the usual way to analyze photoemission data to enhance the electronic dispersions. The spectral weight around $-0.4 \mathrm{eV}$ of the $\varepsilon_{1}$ feature has a very small dispersion, but the spectral weight feature around $-1.4 \mathrm{eV}$ disperses

significantly and seems to reach maxima and minima at reciprocal space positions close to a $(6 \times 6)_{\mathrm{SiC}}$ superperiodicity. This is demonstrated in Figure $2 \mathrm{~g}$ where the vertical dashed lines, marking the $(6 \times 6)_{\mathrm{SiC}}$ zone boundaries perpendicular to the $\Gamma \mathrm{K}$ direction, line up with maxima in the $\varepsilon_{1}$ band intensities. The periodicity of $\varepsilon_{1}$ is further observed in the $E(\mathrm{k})$ dispersions along the $\Gamma \mathrm{M}_{6 \times 6 \mathrm{SiC}}$ directions shown in the Supporting Information (Figure S.2).

The $(6 \times 6)_{S i C}$ periodicity of $\varepsilon_{1}$ is also seen in the constant energy cuts of the electronic structure. Figure 3 shows constant energy cuts at four different energies. The constant energy cuts in Figure $3 b$ and $c$ are near the graphene $K$ point at energies, where $\varepsilon_{1}$ has stronger spectral weight. The spectral weight in these constant energy cuts is not constant in reciprocal space, indicating that the state observed there disperses. Figure $3 \mathrm{~b}$ and c show that the spectral weight of the $\mathrm{p}_{z}$-character of $\varepsilon_{1}$ features is indeed periodic, with a spatial period much larger than the lattice parameter of graphene. This $\mathrm{p}_{z}$-derived state is sensitive to the structural superperiodicity of the $(6 \times 6)_{\mathrm{SiC}}$ reconstruction. Regardless of the precise spatial periodicity (as different commensurate and incommensurate lattice parameters may be defined), if a $\pi$-derived band was sensitive to a strong superperiodic potential, a gap could appear at the Brillouin zone edges of the superperiodicity due to Bragg reflection on them. Such a situation could explain the fact that the spectral weight of the $\varepsilon_{1}$ feature is discontinuous in energy. We first try to figure out the periodicity of this $\mathrm{p}_{z}$-derived state only visible close to the $\mathrm{K}$ point of graphene. $\varepsilon_{1}$ is different from previously observed g1 and g2 states of the buffer layer ${ }^{22}$ as these states are visible in a larger region of the reciprocal space with a

different symmetry (see Supporting Information (Figure S.1). $\varepsilon_{1}$ is compatible with the orientation of $\mathrm{SiC}$ Brillouin zones, with a size close to that of the $(6 \times 6)_{\mathrm{SiC}}$, as 

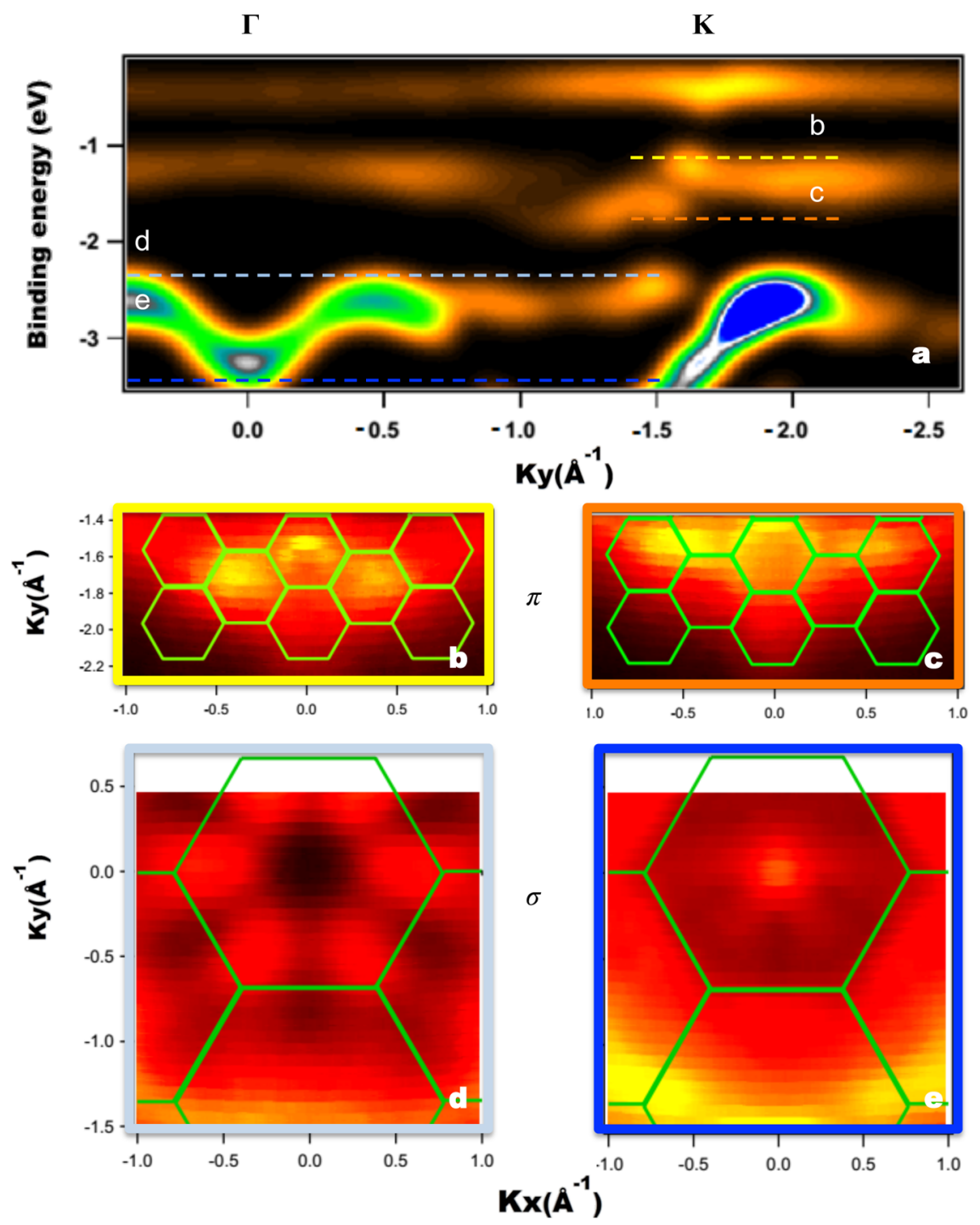

Figure 3. Constant energy cuts through the electronic structure the buffer layer. (a) $E(\mathrm{k})$ dispersion relation along the $\Gamma \mathrm{K}$ direction of graphene. Dashed lines show the energies for the constant energy cuts in $\mathrm{b}-\mathrm{e}$ : (b) $-1.1 \mathrm{eV},(\mathrm{c})-1.7 \mathrm{eV},(\mathrm{d})-2.3 \mathrm{eV}$, and (e) $-3.45 \mathrm{eV}$. The hexagons in the constant energy cuts in panels $\mathrm{b}$ and $\mathrm{c}$ show $(6 \times 6)_{\mathrm{SiC}}$ unit cells. It can be appreciated that the spectral weight follows a periodicity of $(6 \times 6)_{\mathrm{SiC}}$. Panels $\mathrm{d}$ and e show $(\sqrt{3} \times \sqrt{3}) \mathrm{R} 30^{\circ}{ }_{\mathrm{SiC}}$ Brillouin zones corresponding to the symmetry of the spectral weight in this energy range. The Supporting Information includes some line profiles along these figures to make the observed periodicities more explicit.

seen in Figure $3 b$ and c. High spectral weight is not expected in every Brillouin zone as the photoemission intensity is not necessarily periodic, ${ }^{44-46}$ as particularly evident in the paradigmatic case of the $(7 \times 7)$ reconstruction of $\mathrm{Si}(111)$, where the spectral weight of the surface state does not seem to follow the $(7 \times 7)$ periodicity. ${ }^{44}$ This is due to the influence of the structure factor in photoemission, analogous to the situation in X-ray diffraction. The $(6 \times 6)_{\mathrm{SiC}}$ is thus a plausible periodicity according to the constant energy cut, whose atomic origin is to

be identified. It is also important to note that the incommensurate modulation of the buffer electronic density has been shown to be modulated by reciprocal lattice vectors of $\mathrm{SiC}^{43}$

The periodicity of the buffer's $\sigma$ band is different from the $\mathrm{p}_{z}$ bands. Figure $3 \mathrm{~d}$ and e show constant energy cuts at $-2.3 \mathrm{eV}$ and $-3.45 \mathrm{eV}$ near $\Gamma$ (Figure 3a) where $\sigma$ band exists. Figure 3d and e show that the highly dispersive $\sigma$ band is periodic. Any true periodicity must be compatible with the constant energy cuts shown in Figure 3d and e, as they are intercepting the same electronic state, as observed in Figure 3a. It can be observed that the highly dispersive $\sigma$ band follow bigger Brillouin zones than the $\pi$-derived band, oriented as those of $\mathrm{SiC}$ and close to the expected ones for $(\sqrt{3} \times \sqrt{3}) \mathrm{R} 30^{\circ}{ }_{\text {SiC }}$. A similar periodicity has been also observed by other groups. ${ }^{37,38}$

Indications on the atomic origin of the periodicities can be obtained by combining STM and X-ray diffraction. Figure 4a shows the characteristic STM topographic image of the buffer layer. It shows that a honeycomb graphene layer is modulated by an apparent $(6 \times 6)_{\mathrm{SiC}}$ reconstruction (about $1.8 \mathrm{~nm}$ periodicity) that was previously reported for the buffer
layer $^{38,39,4-50}$ and compatible with the incommensurate modulation with a period close to the $(6 \times 6)_{\mathrm{SiC}}$ cell reconstruction observed by X-ray diffraction. ${ }^{43}$ The structural interpretation of the periodicity felt by the $\pi$-derived states is found in Figure $4 a$, where the $(6 \times 6)_{\mathrm{SiC}}$ unit cell indicates a pseudoperiodicity in STM images. STM images also allow to discard a possible band gap opening in the buffer layer due to defects. Figure $4 \mathrm{~b}$ shows a very regular honeycomb lattice with 

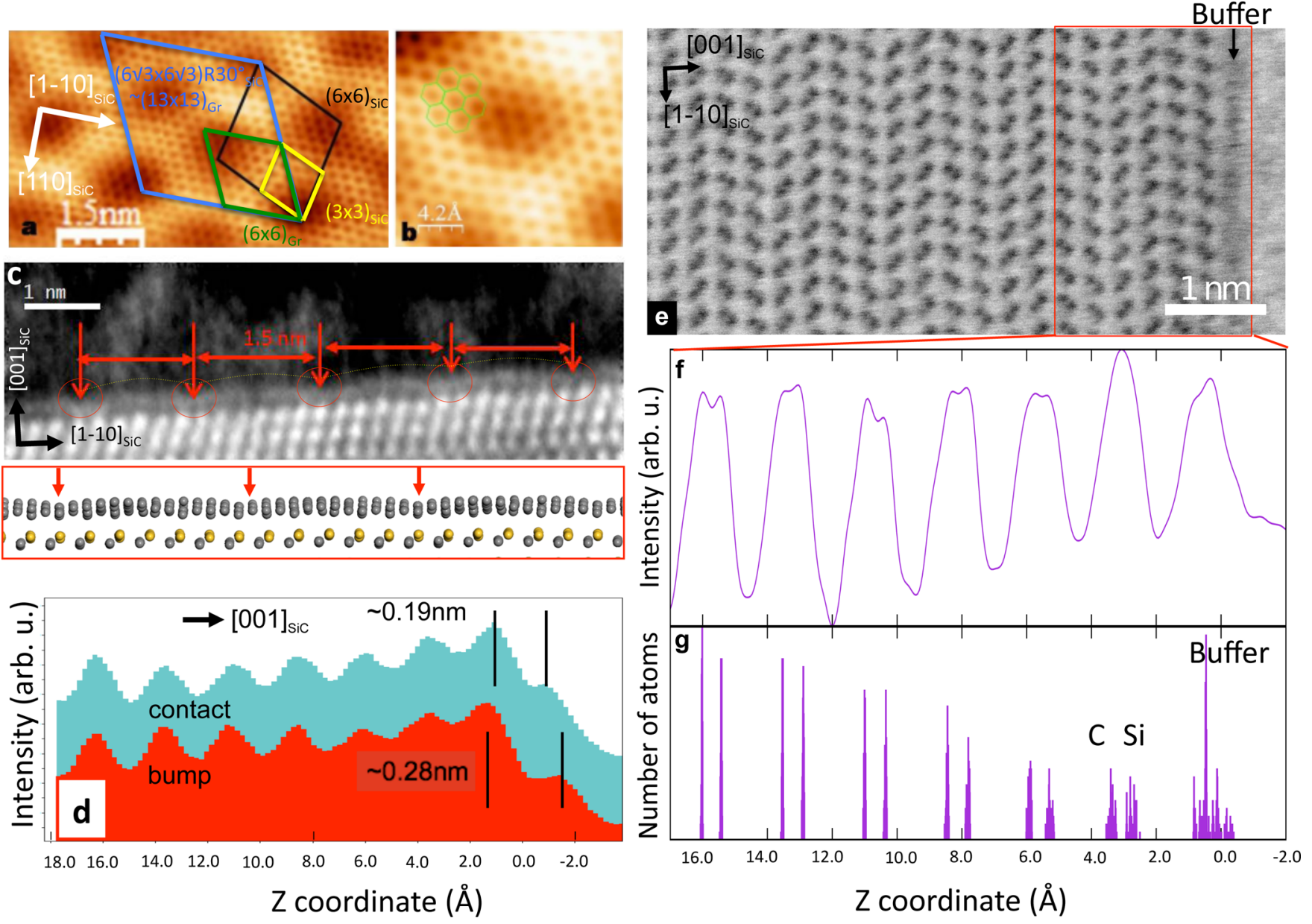

Figure 4. Top and side views of the buffer layer structure. (a) STM image $(I=0.25 \mathrm{nA}, V=-1.5 \mathrm{~V})$ of the buffer layer, showing the quasi- $(6 \times 6)_{\mathrm{SiC}}$ periodicity (black diamond). (b) Close-up on the image shows the graphene honeycomb structure without hexagon-pentagon-heptagon defects. (c) STEM-HAADF image of a cut through the buffer layer graphene-SiC interface. The dotted line is a guide to the eye above the buffer layer to show the bonding points of the buffer layer to the substrate. The overall periodicity between bonding points is $\sim 1.6 \mathrm{~nm}$, compatible with a $(6 \times 6)_{\mathrm{SiC}}$ period. The atomic model in the inset below shows the DFTB relaxed model. (d) Vertical profiles of the interface in c have been obtained by averaging the STEM-HAADF data over several regions where the buffer is attached to the substrate ("contact") and where it is decoupled ("bump"). The red arrows in panel $\mathrm{c}$ indicate where the "contact" profiles were measured. (e) STEM-ABF image resolving Si and C atoms, showing the buffer layer on the right $(z=0)$. (f) Profile obtained from e, showing the contrast distribution for the last six SiC bilayers and the buffer layer. (g) Theoretical number of atoms versus the $z$ coordinate (normal to the surface). The last SiC bilayer and the buffer layer have been indicated.

no evidence for hexagon-pentagon-heptagon defects that were suggested to cause a band gap. ${ }^{51}$ We can also rule out that the buffer layer is uniformly strongly coupled to the $\mathrm{SiC}$ substrate, which would completely suppress the $\pi$-band. Information on the coupling can indeed be provided by cross-sectional STEM images, which are sensitive essentially to the atomic structure, and not convoluted with electronic structure effects like STM. Figure $4 \mathrm{c}$ shows a STEM cross sectional view of the buffer-SiC interface along the crystallo-graphic zone axis $[110]_{\mathrm{SiC}}$. The image was obtained with a $60 \mathrm{keV}$ electron beam to avoid sample damage. Figure $4 c$ corresponds to a high angle annular

dark field (HAADF) collection mode that is sensitive to the heaviest elements. Bright spots are therefore $\mathrm{Si}$ atoms on the substrate and $\mathrm{C}$ atoms in the buffer layer. The crosssectional image conclusively shows that the buffer layer is pinned to the substrate with an apparent periodicity of about 1.5 $\mathrm{nm}$, which corresponds to the projection along the $[1-10]_{\mathrm{SiC}}$ of the $(6 \times$

6) $)_{\mathrm{SiC}}$ lattice parameter. The $1.8 \mathrm{~nm}$ period is in perfect agreement with of the $(6 \times 6)_{\mathrm{SiC}}$ lattice parameter found in STM.
In the place where the buffer carbon is pinned to the $\mathrm{SiC}$, the buffer-substrate distance is reduced to $\sim 1.9 \AA$ (Figure $4 \mathrm{~d}$ ). The pinning distance is very close to the $\mathrm{Si}-\mathrm{C}$ bond length in $\mathrm{SiC}$

(1.89 $\AA$ ) implying a strong $\mathrm{sp}^{3}$ covalent bonding of the buffer, pinning the carbon to $\mathrm{Si}$ in the $\mathrm{SiC}$ interface as suggested by Emtsev et al. ${ }^{22}$ In the decoupled regions, the distance of the buffer layer to the substrate is much larger, $\sim 2.8 \AA$. This distance is only $15 \%$ less than the distance between van der Waals

bonded layers in graphite. The result is that the buffer layer has a total vertical corrugation close to $1 \AA$. Additional structural information on the buffer-SiC interface can be obtained using a different STEM collection mode, that is, annular bright field $(A B F)$, which is sensitive to light elements like carbon. Figure $4 \mathrm{e}$ shows the result of an ABF measurement. Both Si and $\mathrm{C}$ atoms are clearly resolved on the substrate, and since the atomic resolution is very high, it is possible to extract information on the subsurface structural modification when the buffer layer is grown on $\mathrm{SiC}$. To quantify deviations with respect to the bulk structure, we have extracted the intensity profile from Figure $4 \mathrm{e}$ and plotted it in Figure $4 \mathrm{f}$ (the procedure is described in the Methods section). 

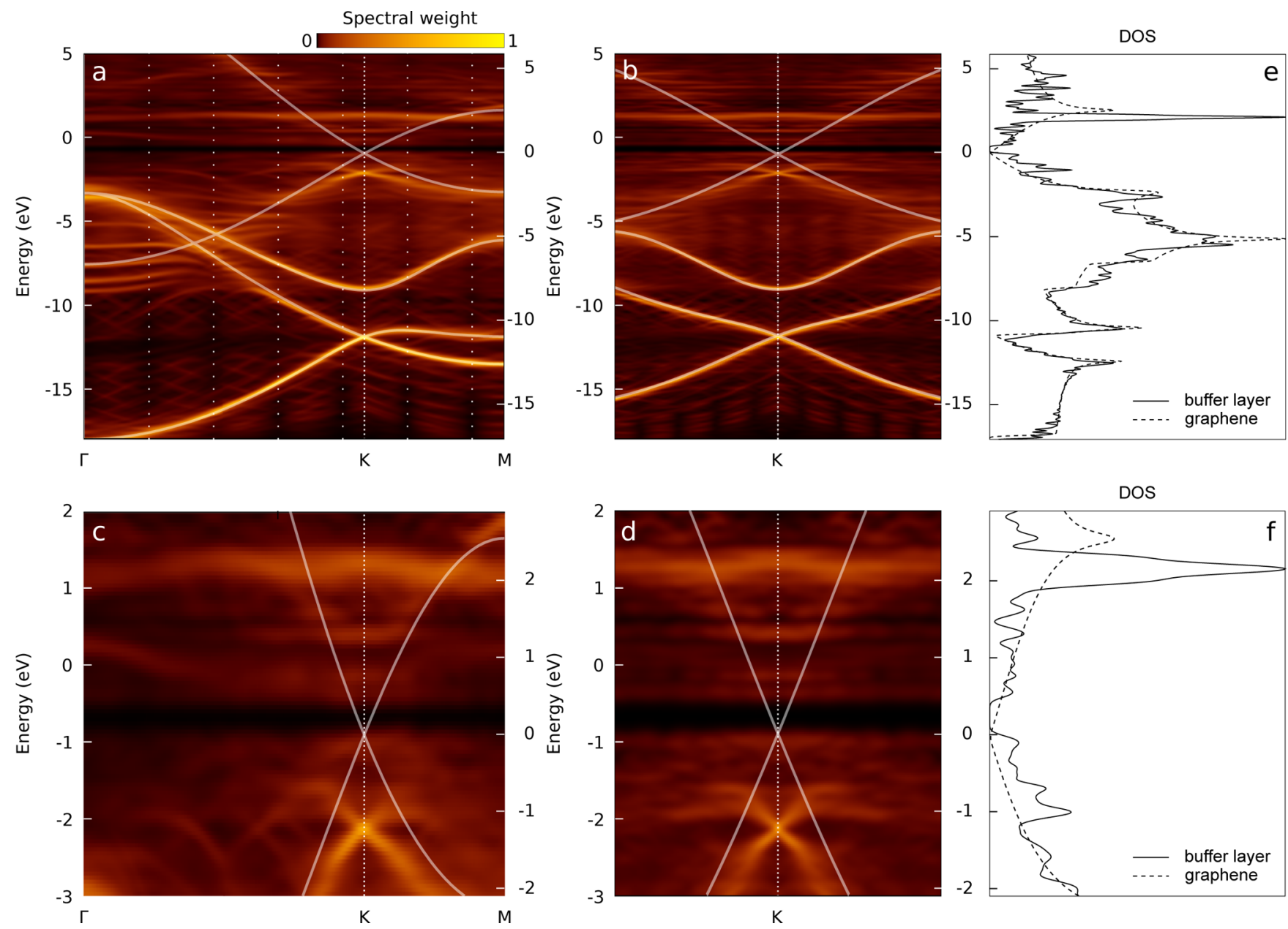

Figure 5. Band structure of the $(6 \sqrt{3} \times 6 \sqrt{ } 3) \mathrm{R} 30^{\circ}{ }_{\mathrm{SiC}}$ buffer layer reconstruction unfolded over $(\mathrm{a}) \Gamma \mathrm{KM}$ and $(\mathrm{b})$ along the perpendicular direction through $\mathrm{K}$ in the extended primitive graphene Brillouin zone (see the reciprocal space scheme in Figure 2a). Color scale represents the spectral weight. The graphene band structure has been superimposed for reference by aligning the deepest $\sigma$ band. Vertical dotted lines in a represent the center of several Brillouin zone of the $(6 \times 6)_{\mathrm{SiC}}$ superperiodicity. An energy broadening of $10 \mathrm{meV}$ has been applied to the bands. Left energy scales refer to the Fermi level as obtained in the supercell model, the right scales to the Fermi level in monolayer graphene. A close-up of the electronic structure near the Fermi level is shown in $\mathrm{c}$ for the $Г \mathrm{KM}$ direction and in $\mathrm{d}$ for the perpendicular direction through $\mathrm{K}$. The density of states for the buffer layer and for graphene is shown in panels e and $\mathrm{f}$, corresponding to the same energy windows used in panels $\mathrm{b}$ and $\mathrm{d}$, respectively.

In this way, we observe a clear separation of the $\mathrm{Si}$ and $\mathrm{C}$ atoms in the $\mathrm{SiC}$ bilayer located at several nanometer from the interface. The distances between the $\mathrm{Si}$ and $\mathrm{C}$ layers are measured at about $60 \mathrm{pm}$ that is in fair agreement with the expected $63 \mathrm{pm}$ along $[001]_{\mathrm{SiC}}$ for a bulk structure. Deviations from the bulk structure become visible for the last three $\mathrm{SiC}$ bilayers and are substantial for the interface $\mathrm{SiC}$ bilayer just below the buffer layer, where the peak separation is not any more visible.

In addition to the structural observations, numerical simulations support both the electronic and structural data described above. We have performed density functional tight binding approach (DFTB) calculations, which combines high accuracy from DFT based parameters with the reduced computational cost of standard tight binding (see the Methods section for more details). This technique is thus advantageous for describing large unit cells with complex bonding reconfiguration such as the $(6 \sqrt{ } 3 \times 6 \sqrt{ } 3) \mathrm{R} 30^{\circ}{ }_{\text {SiC }}$ buffersubstrate interface (that corresponds to a $13 \times 13$ graphene supercell). The resulting optimized atomic positions from the calculations are shown on the inset of Figure 4c. A histogram of the calculated $\mathrm{z}$ atomic coordinates of the atoms is shown in
Figure $4 \mathrm{~g}$ (the $z$-coordinates of all the atoms are summed over the unit cell). These calculations show that $\mathrm{C}$ and Si layers exhibit a certain disorder close to the interface, especially at the last $\mathrm{SiC}$ bilayer, explaining the change of contrast in the experimental profile (Figure 4f). The calculated buffer layer presents a corrugation, with a periodicity and an intensity comparable to the STEM-images of Figure 4c, e, and f. Besides this structural information, the atomic configuration of atoms in the buffer layer indicates that atoms in the decoupled regions are $\mathrm{sp}^{2}$ hybridized, indicating that the buffer layer has the $\pi$-related band of graphene.

The electronic structure derived from this extended supercell model results in highly folded bands. To understand how the electronic structure of the buffer layer is modified with respect to that of graphene, we employed an unfolding postprocessing

which provides a primitive cell effective band structure (see the Methods section for more details). Band unfolding for epitaxial graphene over $\mathrm{SiC}$ has been already presented in the work of Kim and co-workers using a DFT plane waves approach. ${ }^{27}$ Here we use a localized basis set, suitable for larger clusters and providing an easier interpretation of the states by projecting on a subset of orbitals or atoms. We considered the spectral 
contribution for sole carbon atoms at the buffer layer, which represents a good approximation for the limited penetration of photons in ARPES. Figure 5 shows the band structure of the $(6 \sqrt{ } 3 \times 6 \sqrt{3}) \mathrm{R}_{30}{ }^{\circ} \mathrm{SiC}$ reconstruction unfolded over the graphene Brillouin zone as well as the density of states. The perfect graphene band structure has been superimposed by aligning the deepest $\sigma$ band (white continuous line). The left energy scale refers to the Fermi level as obtained in the supercell model for the buffer layer, the right scale to the Fermi level in perfect graphene. Note that $\mathrm{SiC}$ has an intrinsic polarization, which, in the absence of dipole corrections, gets equilibrated in supercell calculations by a surface charge overestimation. This is due to the periodic boundary condition that is imposed to the potential in the supercell. This effect leads to a spurious upper shift of the Fermi level at the buffer layer. To compare with experimental data it is therefore more pertinent to refer all calculated energies from the Dirac point energy of the perfect graphene.

In agreement with previous results, we observe that $\sigma$ bands in the buffer layer and in graphene disperse similarly. The translation symmetry breaking in the buffer supercell is responsible for a residual spectral weight at the unfolded $\sigma$ bands, which is further modulated by the apparent $(6 \times 6)_{\mathrm{SiC}}$ reconstruction. This effect is clearly visible in Figure 5a, where the center of several Brillouin zones of the $(6 \times 6)_{\mathrm{SiC}}$ reconstruction has been indicated with vertical dotted lines.

The $\pi$ band of the buffer layer can also be identified, whose dispersion is significantly different to that of graphene. The $\pi$ band splits at the $\Gamma$ point due to rehybridization with $\mathrm{SiC}$ bands (for $\mathrm{SiC}$ allotropes band structures and surface states see for instance ref 52). For higher wave numbers, we observe a maximum spectral weight at $\mathrm{K}$ where a " $\mathrm{X}$ "-shaped feature arises about $1.2 \mathrm{eV}$ below the graphene Dirac cone. A similar remarkably intense " $\mathrm{X}$ "-shaped feature is also experimentally observed both in the buffer and in the graphene monolayer (see Figure 1).

Above the Dirac cone position, the system presents a calculated $0.4 \mathrm{eV}$ band gap, clearly visible as a dark line in the band structure but also in the density of states. Note that DFTB based calculations usually underestimate the band gap values and $0.4 \mathrm{eV}$ might be seen as a lower bound. As mentioned previously, the position of the Fermi level in the calculation is overestimated (it is located $1 \mathrm{eV}$ above the Dirac point energy of the graphene, it is in the conduction band of the buffer layer). Nevertheless, the real position of the Fermi level can be estimated with the help of experimental observations. It has

been reported that graphene on top of the buffer layer is slightly $\mathrm{n}$ doped leading to a Fermi level several hundreds of $\mathrm{meV}$ above the graphene Dirac cone. This provides a constraint on the

buffer layer Fermi level position which therefore lies within the computed band gap indicating the semiconducting character of the buffer layer.

The weakly dispersive bands observed experimentally between the intense " $X$ "-shaped feature and the band gap also appear in these calculations. In the ARPES measurement, these states have maxima roughly distributed along a parabola (see Figure $2 \mathrm{~d}, \mathrm{~g}$ ) and have a lower velocity than the dispersion of isolated graphene would predict. The calculated bands (Figure 5c,d and Figure S.4) have more complicated and sharper details, notably due to lack of disorder, incommensurability, and other broadening source that are not included in the calculations. Nevertheless, the weakly dispersive character and the appearance of discontinuous contrast in the spectral weight are also observed in the calculation. The comparison of the DOS calculated for the graphene and the buffer layer is also of interest. In the occupied domain, both DOS match rather well, except in the range from 0 to $-1 \mathrm{eV}$. In this energy domain, narrow peaks are only present for the buffer layer. Their energies are very comparable with the experimentally reported maxima energies of -0.4 and $-1.4 \mathrm{eV}$ (Figure $2 \mathrm{~d}$ ), notably keeping in mind that the Fermi level in the calculated DOS lie several hundred of $\mathrm{meV}$ above the zero. Such electronic states may be related to local orders in the $(6 \sqrt{3} \times 6 \sqrt{ } 3) \mathrm{R} 30^{\circ}$ supercell and possibly with the apparent $6 \times 6$ reconstruction. Such structural periodicities are known to be present as measured by several

different structural techniques STM, TEM, LEED, or XRD (Figure 4), corresponding to a strong interaction between the buffer layer and the underlying substrate, evident from the formation of out-of-plane covalent bonds. We thus conclude that not only the $\sigma$ bands of graphene are affected by the structural periodicities of $\mathrm{SiC}$ but also the $\pi$ bands, which are at the origin the band gap opening.

In conclusion, we have analyzed the band gap opening in the graphene buffer layer by employing a complementary set of experimental and theoretical approaches that include STM, STEM, and ARPES with DFTB based calculations. The combined results from this approach allow us to conclude that the gap around the Dirac point does not originate from a significant density of hexagon-pentagon-heptagon defects or the complete pinning of the buffer layer to the substrate. As suggested by STM images, cross-sectional STEM demonstrates that the buffer layer is only pinned at some precise locations. In between these pinning regions, separated by $1.5 \mathrm{~nm}$, the buffer shows areas of decoupled graphene, with $\mathrm{sp}^{2}$ hybridization. Nonetheless, these decoupled areas exhibit a band gap, as the whole buffer layer system. The main result is that the buffer layer $\pi$ band shows band gaps aligned with the substrate $6 \times 6$ nanometric superperiodicity. The induced band gap in this periodic graphene system resembles the gap opening in graphene nanoribbons. ${ }^{19}$ However, the difference between these two systems is that in graphene nanoribbons, the periodicity plays no role in the band gap opening. In the buffer layer, the situation is completely different. The band gap opens in the $\pi$ band due to a nanoscale superperiodicity. These results indicate new ways of controlling graphene's band structure simply by transferring or growing graphene on top of self-organized or nanostructured systems.

METHODS. Experimental Details. Graphene samples were prepared from a n-doped $4 \mathrm{H}-\mathrm{SiC}(0001)$ substrate in a controlled silicon sublimation furnace. ${ }^{53}$ The semiconducting buffer layer graphene in this letter was prepared using the same procedure as in Nevius et al. ${ }^{28}$

Graphitized samples were transported in air and thermally annealed at $500 \mathrm{~K}$ in UHV prior to measurements. ARPES measurements were carried out at the CASSIOPEE beamline at SOLEIL synchrotron radiation source. The beamline is well equipped with a Peterson PGM monochromator with an energy resolution $E / \Delta E$ of 25000 for energies below $100 \mathrm{eV}$. The detector is a Scienta R4000 detector with a base resolution of $\Delta E<1 \mathrm{meV}$. Binding energies are referenced to the Fermi level of a metal in electrical contact with the sample. The photon energy used for the constant energy cuts in Figure 3 was $70 \mathrm{eV}$. The photon energy for Figure $1 \mathrm{a}$ and $\mathrm{b}$ was 70 and $150 \mathrm{eV}$, respectively. The STM experiments were performed with a variable temperature (VTSTM) from Omicron. Bias voltages were applied to the grounded PtIr tip. STEM-EELS 
measurements were carried out using a STEM NION microscope and an in-house modified GATAN spectrometer. To limit the electron beam damage, most of the experiments were done with $60 \mathrm{keV}$ electron beam, unless otherwise specified. Atomic resolution was obtained in STEM mode by correcting the $3 / 5$ order spherical aberration of the objective lens. Figure $4 \mathrm{c}$ is a high angle annular dark field (HAADF) image made by collecting scattered electrons with angles between 80 and 180 mrads. Figure $4 \mathrm{e}$ is an STEM-ABF (annular bright field) measurement performed at $200 \mathrm{keV}$ with a resolution close to $70 \mathrm{pm}$. The intensity profile from this figure has been obtained by integrating over $5 \mathrm{~nm}$ in a direction parallel to the surface after applying a deconvolution filter to sharpen the atomic columns (such integration increases the signal-to-noise ratio and it is enough to average over several reconstruction periods). To compare with the STEM-HAADF profile (Figure 4d), the STEM-ABF profile contrast has been reversed (Figure 4f). Sample preparation for STEM is described elsewhere. ${ }^{19}$

Computational Details. We consider a commensurate $(6 \sqrt{ } 3$ $\times 6 \sqrt{ } 3) \mathrm{R} 30^{\circ} \mathrm{SiC}$ supercell where the silicon terminated upper $\mathrm{SiC}$ surface matches the carbon buffer layer's area (corresponding to a graphene $13 \times 13$ supercell). To avoid spurious interactions from the bottom surface, we use a 12 atomic layer model of the $\mathrm{SiC}$ substrate (one $\mathrm{SiC}-6 \mathrm{H}$ unit cell along the $\mathrm{c}$ direction) with dangling bonds at the lower carbon surface saturated by hydrogen atoms. The total size of the system was 1742 atoms. All calculations have been performed using the DFTB scheme in its self-consistent extension, ${ }^{54}$ as implemented in the $\mathrm{dftb}+$ code. $^{55}$ We employed the pbc- $0-3$ parameter set that has been demonstrated to provide a good representation of structural and electronic properties for $\mathrm{SiC}$ in respect to DFT-LDA ${ }^{56}$ (band dispersions are only slightly underestimated). Atomic positions have been relaxed until forces were below $10^{-4} \mathrm{eV} / \AA ̊$ on each atom. To compare the buffer layer electronic band structure with respect to the perfect graphene bands, we employed the unfolding postprocessing method developed by Lee and coworkers. ${ }^{57,58}$ This technique, originally derived for Wannier and linear combination of atomic orbitals basis functions, can be directly employed in the framework of DFTB. ${ }^{59}$ In the representation of Figure 5, an energy broadening of $10 \mathrm{meV}$ has been applied to the bands.

ACKNOWLEDGMENTS This work was supported by the French Agence Nationale de la Recherche (ANR), project CoRiGraph, ref ANR-12-BS04-0017 and by the French American Cultural Exchange council through a Partner University Fund project. The authors acknowledge support from CASSIOPEE beamline staff and F. Nicolas from SOLEIL synchrotron. C.B. acknowledges partial financial support from the EU graphene Flagship program. Financial support was provided to W.d.H. and C.B. by the AFSOR under Grant No. FA9550-13-0217 and NSF No. 1506006. E.H.C. would like to acknowledge support from the NSF under Grant No. DMR-1401193.

\section{REFERENCES}

(1) Baringhaus, J.; Ruan, M.; Edler, F.; Tejeda, A.; Sicot, M.; TalebIbrahimi, A.; Li, A.-P.; Jiang, Z.; Conrad, E. H.; Berger, C.; Tegenkamp, C.; de Heer, W. A. Nature 2014, 506, 349-354.

(2) Sprinkle, M.; Siegel, D.; Hu, Y.; Hicks, J.; Tejeda, A.; TalebIbrahimi, A.; Le Févre, P.; Bertran, F.; Vizzini, S.; Enriquez, H.; Chiang, S.; Soukiassian, P.; Berger, C.; de Heer, W. A.; Lanzara, A.; Conrad, E. H. Phys. Rev. Lett. 2009, 103, 226803.

(3) Dlubak, B.; Martin, M.-B.; Deranlot, C.; Servet, B.; Xavier, S.; Mattana, R.; Sprinkle, M.; Berger, C.; De Heer, W. A.; Petroff, F.; Anane, A.; Seneor, P.; Fert, A. Nat. Phys. 2012, 8, 557-561.

(4) Ruffieux, P.; Wang, S.; Yang, B.; Sanchez, C.; Liu, J.; Dienel, T.; Talirz, L.; Shinde, P.; Pignedoli, C. A.; Passerone, D.; Dumslaff, T.; Feng, X.; Müllen, K.; Fasel, R. Nature 2016, 531, 489-492.

(5) Berger, C.; Song, Z.; Li, T.; Li, X.; Ogbazghi, A. Y.; Feng, R.; Dai, Z.; Marchenkov, A. N.; Conrad, E. H.; First, P. N.; De Heer, W. A. J. Phys. Chem. B 2004, 108, 19912-19916.

(6) Balog, R.; Jørgensen, B.; Nilsson, L.; Andersen, M.; Rienks, E.; Bianchi, M.; Fanetti, M.; Laegsgaard, E.; Baraldi, A.; Lizzit, S.; Sljivancanin, Z.; Besenbacher, F.; Hammer, B.; Pedersen, T. G.; Hofmann, P.; Hornekaer, L. Nat. Mater. 2010, 9, 315-319.

(7) Castellanos-Gomez, A.; Wojtaszek, M.; Arramel; Tombros, N.; Van Wees, B. J. Small 2012, 8, 1607-1613.

(8) Matis, B. R.; Burgess, J. S.; Bulat, F. A.; Friedman, A. L.; Houston,

B. H.; Baldwin, J. W. ACS Nano 2012, 6, 17-22.

(9) Zhang, W.; Lin, C.-T.; Liu, K. K.; Tite, T.; Su, C. Y.; Chang, C. H.; Lee, Y. H.; Chu, C. W.; Wei, K. H.; Kuo, J. L.; Li, L. J. ACS Nano 2011, 5, 7517-7524.

(10) Biel, B.; Triozon, F.; Blase, X.; Roche, S. Nano Lett. 2009, 9, 2725-2729.

(11) Haberer, D.; Vyalikh, D. V.; Taioli, S.; Dora, B.; Farjam, M.; Fink, J.; Marchenko, D.; Pichler, T.; Ziegler, K.; Simonucci, S.; et al. Nano Lett. 2010, 10, 3360-3366.

(12) Wang, F.; Liu, G.; Rothwell, S.; Nevius, M.; Tejeda, A.; TalebIbrahimi, A.; Feldman, L. C.; Cohen, P. I.; Conrad, E. H. Nano Lett. 2013, 13, 4827-4832.

(13) Jung, J.; DaSilva, A.; MacDonald, A. H.; Adam, S. Nat. Commun. 2015, 6, 6308 .

(14) Kim, K.; Yankowitz, M.; Fallahazad, B.; Kang, S.; Movva, H. C. P.; Huang, S.; Larentis, S.; Corbet, C. M.; Taniguchi, T.; Watanabe, K.; Banerjee, S. K.; Leroy, B. J.; Tutuc, E. Nano Lett. 2016, 16, 19891995.

(15) Celis, A.; Nair, M. N.; Taleb-Ibrahimi, A.; Conrad, E. H.; Berger, C.; de Heer, W. A.; Tejeda, A. J. Phys. D: Appl. Phys. 2016, 49, 143001 and references therein..

(16) Han, M. Y.; Brant, J. C.; Kim, P. Phys. Rev. Lett. 2010, 104, 056801. 
(17) Sprinkle, M.; Ruan, M.; Hu, Y.; Hankinson, J.; Rubio-Roy, M.; Zhang, B.; Wu, X.; Berger, C.; de Heer, W. A. Nat. Nanotechnol. 2010, $5,727-731$.

(18) Hicks, J.; Tejeda, A.; Taleb-Ibrahimi, A.; Nevius, M. S.; Wang, F.; Shepperd, K.; Palmer, J.; Bertran, F.; Le Fèvre, P.; Kunc, J.; de Heer, W. A.; Berger, C.; Conrad, E. H. Nat. Phys. 2012, 9, 49-54.

(19) Palacio, I.; Celis, A.; Nair, M. N.; Gloter, A.; Zobelli, A.; Sicot, M.; Malterre, D.; Nevius, M. S.; de Heer, W. A.; Berger, C.; Conrad, E. H.; Taleb-Ibrahimi, A.; Tejeda, A. Nano Lett. 2015, 15, 182.

(20) Varchon, F.; Feng, R.; Hass, J.; Li, X.; Nguyen, B. N.; Naud, C.; Mallet, P.; Veuillen, J.-Y.; Berger, C.; Conrad, E. H.; Magaud, L. Phys. Rev. Lett. 2007, 99, 126805.

(21) Huang, H.; Chen, W.; Chen, S.; Wee, A. T. S. ACS Nano 2008, 2, 2513-2518.

(22) Emtsev, K. V.; Speck, F.; Seyller, T.; Ley, L.; Riley, J. D. Phys. Rev. B: Condens. Matter Mater. Phys. 2008, 77, 155303.

(23) Ohta, T.; Bostwick, A.; McChesney, J. L.; Seyller, T.; Horn, K.; Rotenberg, E. Phys. Rev. Lett. 2007, 98, 206802.

(24) Riedl, C.; Coletti, C.; Iwasaki, T.; Zakharov, A. A.; Starke, U. Phys. Rev. Lett. 2009, 103, 246804.

(25) Ristein, J.; Mammadov, S.; Seyller, Th. Phys. Rev. Lett. 2012, 108, 246104.

(26) Mattausch, A.; Pankratov, O. Phys. Status Solidi B 2008, 245, 1425.

(27) Kim, S.; Ihm, J.; Choi, H.; Son, Y.-W. Phys. Rev. Lett. 2008, 100, 176802.

(28) Nevius, M. S.; Conrad, M.; Wang, F.; Celis, A.; Nair, M. N.; Taleb-Ibrahimi, A.; Tejeda, A.; Conrad, E. H. Phys. Rev. Lett. 2015, $115,136802$.

(29) Zhou, S. Y.; Gweon, G.-H.; Graf, J.; Fedorov, A. V.; Spataru, C. D.; Diehl, R. D.; Kopelevich, Y.; Lee, D.-H.; Louie, S. G.; Lanzara, A. Nat. Phys. 2006, 2, 595-599.

(30) Zugarramurdi, A.; Debiossac, M.; Lunca-Popa, P.; Mayne, A. J.; Momeni, A.; Borisov, A. G.; Mu, Z.; Roncin, P.; Khemliche, H. Appl. Phys. Lett. 2015, 106, 101902.

(31) Lampin, E.; Priester, C.; Krzeminski, C.; Magaud, L. J. Appl. Phys. 2010, 107, 103514.

(32) Peng, X.; Ahuja, R. Nano Lett. 2008, 8, 4464.

(33) Inoue, M.; Kageshima, H.; Kangawa, Y.; Kakimoto, K. Phys. Rev. B: Condens. Matter Mater. Phys. 2012, 86, 085417.

(34) Mattausch, A.; Pankratov, O. Phys. Rev. Lett. 2007, 99, 076802.

(35) Mallet, P.; Varchon, F.; Naud, C.; Magaud, L.; Berger, C.; Veuillen, J.-Y. Phys. Rev. B: Condens. Matter Mater. Phys. 2007, 76 (4), 041403.

(36) Chen, W.; Xu, H.; Liu, L.; Gao, X.; Qi, D.; Peng, G.; Tan, S. C.; Feng, Y.; Loh, K. P.; Wee, A. T. S. Surf. Sci. 2005, 596, 176-186.

(37) Riedl, C.; Starke, U.; Bernhardt, J.; Franke, M.; Heinz, K. Phys. Rev. B: Condens. Matter Mater. Phys. 2007, 76, 245406.

(38) Rutter, G. M.; Guisinger, N. P.; Crain, J. N.; Jarvis, E. a. a.; Stiles, M. D.; Li, T.; First, P. N.; Stroscio, J. A. Phys. Rev. B: Condens. Matter Mater. Phys. 2007, 76, 235416.

(39) Owman, F.; Martensson, P. Surf. Sci. 1996, 369, 126.

(40) Forbeaux, I.; Themlin, J. M.; Debever, J. M. Phys. Rev. B: Condens. Matter Mater. Phys. 1998, 58, 16396.

(41) Seyller, T.; Emtsev, K. V.; Gao, K.; Speck, F.; Ley, L.; Tadich, A.; Broekman, L.; Riley, J. D.; Leckey, R. C. G.; Rader, O.; Varykhalov, A.; Shikin, A. M. Surf. Sci. 2006, 600, 3906.

(42) Goler, S.; Coletti, C.; Piazza, V.; Pingue, P.; Colangelo, F.; Pellegrini, V.; Emtsev, K. V.; Forti, S.; Starke, U.; Beltram, F.; Heun, S. Carbon 2013, 51, 249-254.

(43) Conrad, M.; Wang, F.; Nevius, M. S.; Jinkins, K.; Celis, A.; Narayanan Nair, M. N.; Tejeda, A.; Garreau, Y.; Vlad, A.; Coati, A.; Miceli, P. F.; Conrad, E. H.; Taleb-Ibrahimi, A. Nano Lett. 2017, 17, 341.

(44) Losio, R.; Altmann, K. N.; Himpsel, F. J. Phys. Rev. B: Condens. Matter Mater. Phys. 2000, 61, 10845-10853.

(45) Stoffel, M.; Tejeda, A.; Kierren, B.; Nicolaou, A.; Le Fevre, P.; Bertran, F.; Taleb-Ibrahimi, A.; Malterre, D.; Fagot-Revurat, Y. Phys. Rev. B: Condens. Matter Mater. Phys. 2012, 86, 195438.
(46) Srour, W.; Tejeda, A.; Nicolaou, A.; Le Fèvre, P.; Bertran, F.; Taleb-Ibrahimi, A.; Malterre, D. J. Electron Spectrosc. Relat. Phenom. 2012, 185, 441-447.

(47) Varchon, F.; Mallet, P.; Veuillen, J.-Y.; Magaud, L. Phys. Rev. B: Condens. Matter Mater. Phys. 2008, 77, 235412.

(48) Martensson, P.; Owman, F.; Johansson, L. I. Phys. Status Solidi B 1997, 202, 501-528.

(49) Li, L.; Tsong, I. S. T. Surf. Sci. 1996, 351, 141-148.

(50) Lauffer, P.; Emtsev, K. V.; Graupner, R.; Seyller, T.; Ley, L.; Reshanov, S. A.; Weber, H. B. Phys. Rev. B: Condens. Matter Mater. Phys. 2008, 77, 155426.

(51) Qi, Y.; Rhim, S. H.; Sun, G. F.; Weinert, M.; Li, L. Phys. Rev. Lett. 2010, 105, 085502.

(52) Sołtys, J.; Piechota, J.; Łopuszyński, M.; Krukowski, S. New J. Phys. 2010, 12, 043024.

(53) De Heer, W. A.; Berger, C.; Ruan, M.; Sprinkle, M.; Li, X.; Hu, Y.; Zhang, B.; Hankinson, J.; Conrad, E. H. Proc. Natl. Acad. Sci. U. S. A. 2011, 108, 16900-16905.

(54) Elstner, M.; Porezag, D.; Jungnickel, G.; Elsner, J.; Haugk, M.; Frauenheim, T.; Suhai, S.; Seifert, G. Phys. Rev. B: Condens. Matter Mater. Phys. 1998, 58, 7260.

(55) Aradi, A.; Hourahine, B.; Frauenheim, Th. J. Phys. Chem. A 2007, 111, 5678.

(56) Rauls, E.; Elsner, J.; Gutierrez, R.; Frauenheim, T. Solid State Commun. 1999, 111, 459-464.

(57) Ku, W.; Berlijn, T.; Lee, C.-C. Phys. Rev. Lett. 2010, 104, 216401.

(58) Lee, C.-C.; Yamada-Takamura, Y.; Ozaki, T. J. Phys.: Condens. Matter 2013, 25, 345501.

(59) Farjam, M. J. Phys.: Condens. Matter 2014, 26, 155502. 